\title{
Synthesis of ZnO Crystals with Octahedral Morphology through Thermal Evaporation Technique in Air
}

\author{
Min-Sung $\mathrm{Kim}^{1}$ and Geun-Hyoung Lee ${ }^{2, *}$ \\ ${ }^{1}$ Department of Information \& Communications Engineering, Tong-Myong University, \\ 179 Sinseonno, Nam-gu, Busan 608-711, Republic of Korea \\ ${ }^{2}$ Department of Materials \& Components Engineering, Dong-eui University, \\ 995 Eomgwangno, Busanjin-gu, Busan 614-714, Republic of Korea
}

\begin{abstract}
$\mathrm{ZnO}$ crystals with octahedral morphology were synthesized in large quantity using a simple thermal evaporation of $\mathrm{ZnF}_{2}$ powder in air. No catalysts and impurities were not used in the synthetic process. Scanning electron microscopy image showed that the synthesized $\mathrm{ZnO}$ crystals were of well-defined octahedral shape with smooth surfaces. X-ray diffraction data revealed that the octahedral-shaped $\mathrm{ZnO}$ crystals had a hexagonal wurtzite structure. As the synthetic temperature increased, the crystal size increased without altering the octahedral morphology. Energy dispersive X-ray spectroscopy spectrum confirmed the high purity of the $\mathrm{ZnO}$ crystals. Two emission peaks at around $430 \mathrm{and} 460 \mathrm{~nm}$ was observed in the cathodoluminescence spectrum of the octahedral-shaped ZnO crystals. [doi:10.2320/matertrans.M2013402]
\end{abstract}

(Received October 31, 2013; Accepted January 20, 2014; Published March 7, 2014)

Keywords: zinc oxide, octahedron, zinc fluoride, oxidation, thermal evaporation

\section{Introduction}

Semiconductor nanocrystals exhibit unique electrical or optical properties distinct from those of bulk materials because of the high surface to volume ratio. Among them, $\mathrm{ZnO}$ has attracted attention due to its wide band gap of $3.37 \mathrm{eV}$ and large exciton binding energy of $60 \mathrm{meV}$, which makes it a potential material for applications in optoelectronic devices such as ultravilolet (UV) emitting devices. So far, $\mathrm{ZnO}$ nanocrystals with diverse morphologies including wires, ${ }^{1)}$ belts, ${ }^{2)}$ tubes, ${ }^{3)}$ combs $^{4)}$ and tetrapods ${ }^{5)}$ have been synthesized through various synthetic methods such as metal-organic chemical vapor deposition, ${ }^{6}$ laser ablation, ${ }^{7)}$ thermal evaporation, ${ }^{8)}$ hydrothermal method ${ }^{9)}$ and sol-gel method. ${ }^{10)}$ Among others, thermal evaporation is relatively simple and very cost technique. So, thermal evaporation technique has been widely used to synthesize nanocrystals.

On the other hand, even though many efforts have been made to synthesize $\mathrm{ZnO}$ nanocrystals with various morphologies because the properties of nanocrystals also depend on the morphology, it is still a challenge to synthesize $\mathrm{ZnO}$ nanocrystals with high symmetry such as tetrahedron and octahedron.

In this paper, we report the synthesis of $\mathrm{ZnO}$ crystals with octahedral morphology via thermal evaporation of appropriate source material in air. To my knowledge, this is the first report on the fabrication of octahedral-shaped $\mathrm{ZnO}$ crystals through evaporation-condensation process like thermal evaporation. In particular, there is a controversial issue about the crystal structure of nuclei for the formation of $\mathrm{ZnO}$ crystals with tetrapod morphology. One is based on the formation of the octahedral nuclei with zinc blend structure in the initiation of wurtzite $\mathrm{ZnO}$ tetrapods. ${ }^{11)}$ The other is that wurtzite $\mathrm{ZnO}$ tetrapods grow from octahedral nuclei with wurtzite structure. ${ }^{12)}$ In the present study, it also was found which crystal structure $\mathrm{ZnO}$ octahedra take.

*Corresponding author, E-mail: ghl@deu.ac.kr

\section{Experimental Procedure}

$\mathrm{ZnO}$ crystals were synthesized via thermal evaporation of $\mathrm{ZnF}_{2}$ powder in the range of $1000-1300^{\circ} \mathrm{C}$ in air atmosphere. $\mathrm{ZnF}_{2}$ powder was put in an alumina crucible and the alumina crucible was inserted into the center of the furnace. Then the furnace was heated to the evaporation temperature with a heating rate of $10^{\circ} \mathrm{C} / \mathrm{min}$. The evaporation and oxidation of $\mathrm{ZnF}_{2}$ powder were carried out at the temperature for $1 \mathrm{~h}$. After the oxidation, the furnace was turned off and cooled down to room temperature. A white colored product was formed in the crucible and was collected for the analysis and characterization.

The morphology of the product was observed by field emission scanning electron microscope (FESEM) operated at a voltage of $15 \mathrm{kV}$. The crystal structure was determined by X-ray diffractometer (XRD) with $\mathrm{Cu} \mathrm{K} \alpha$ radiation $(\lambda=$ $0.154 \mathrm{~nm}$ ) operated at a power of $40 \mathrm{kV}$ and $30 \mathrm{~mA}$. The components and composition of the product were studied by energy dispersive X-ray (EDX) spectroscopy. The cathodoluminescence (CL) spectrum was taken by CL spectroscopy at room temperature.

\section{Results and Discussions}

The morphology of the product was observed by FESEM. Figures 1(a) and 1(b) show the low and high magnification SEM images of the product. From these images, it can be found that the product is composed of micro-particles with an octahedron morphology. The octahedron shape is clearly shown in the high magnification SEM image.

The crystallographic structure of the particles with octahedral morphology was studied through XRD measurement. The XRD pattern of the particles with octahedron morphology is shown in Fig. 2(a). All diffraction peaks are in accordance with standard peaks of $\mathrm{ZnO}$ with a hexagonal wurtzite structure. The peaks at $31.8^{\circ}, 34.4^{\circ}, 36.2^{\circ}, 47.5^{\circ}$, $56.6^{\circ}$ can be indexed to $(10 \overline{1} 0),(0002),(10 \overline{1}),(10 \overline{1} 2)$ and 

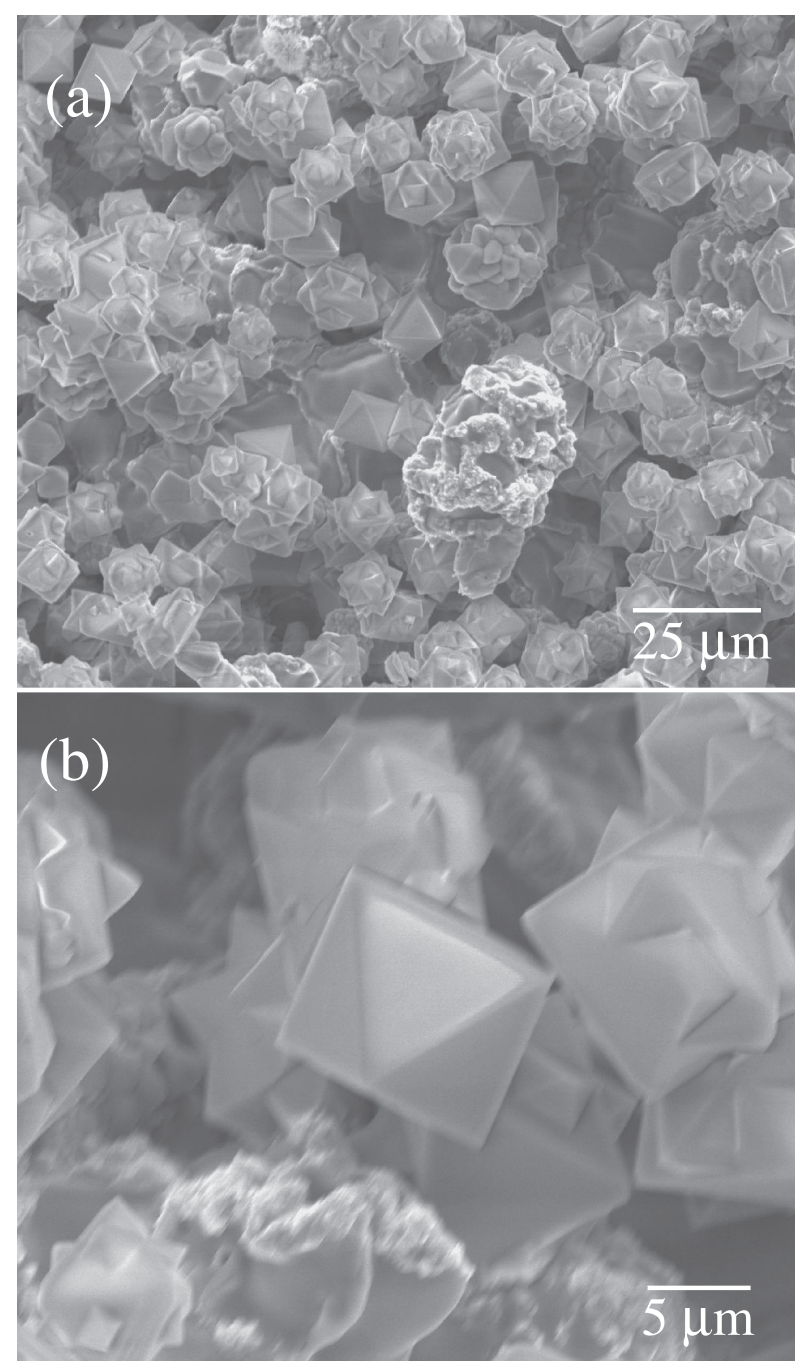

Fig. 1 (a) Low and (b) high-magnification SEM images of the product prepared via thermal evaporation of $\mathrm{ZnF}_{2}$ powder.
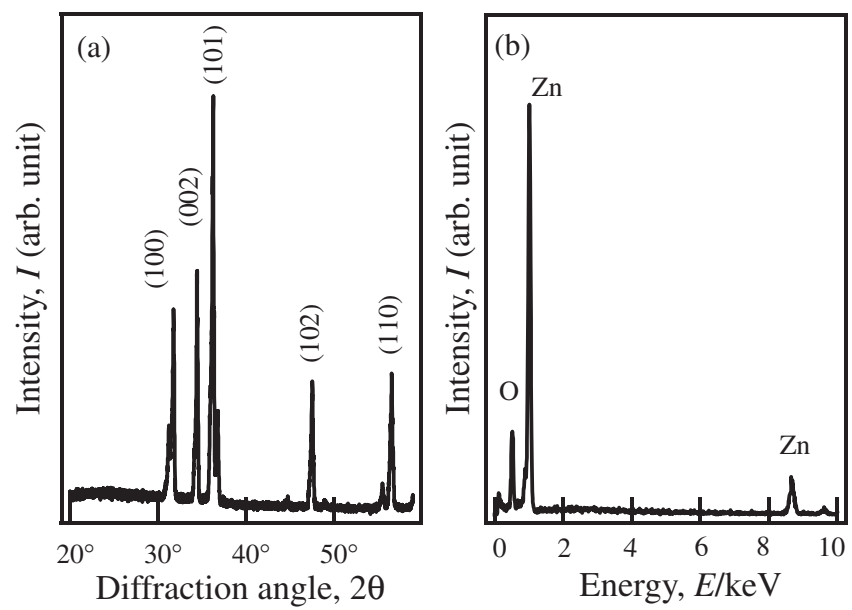

Fig. 2 (a) XRD patterns and (b) EDX spectrum of the products prepared via thermal evaporation of $\mathrm{ZnF}_{2}$ powder.

(11) 0 ) lattice planes of wurtzite structure of $\mathrm{ZnO}$ with the lattice constants of $a=b=0.324 \mathrm{~nm}$ and $c=0.520 \mathrm{~nm}$ (JCPDS 36-1451). This indicates that the octahedralshaped particles are $\mathrm{ZnO}$ with wurtzite crystal structure.
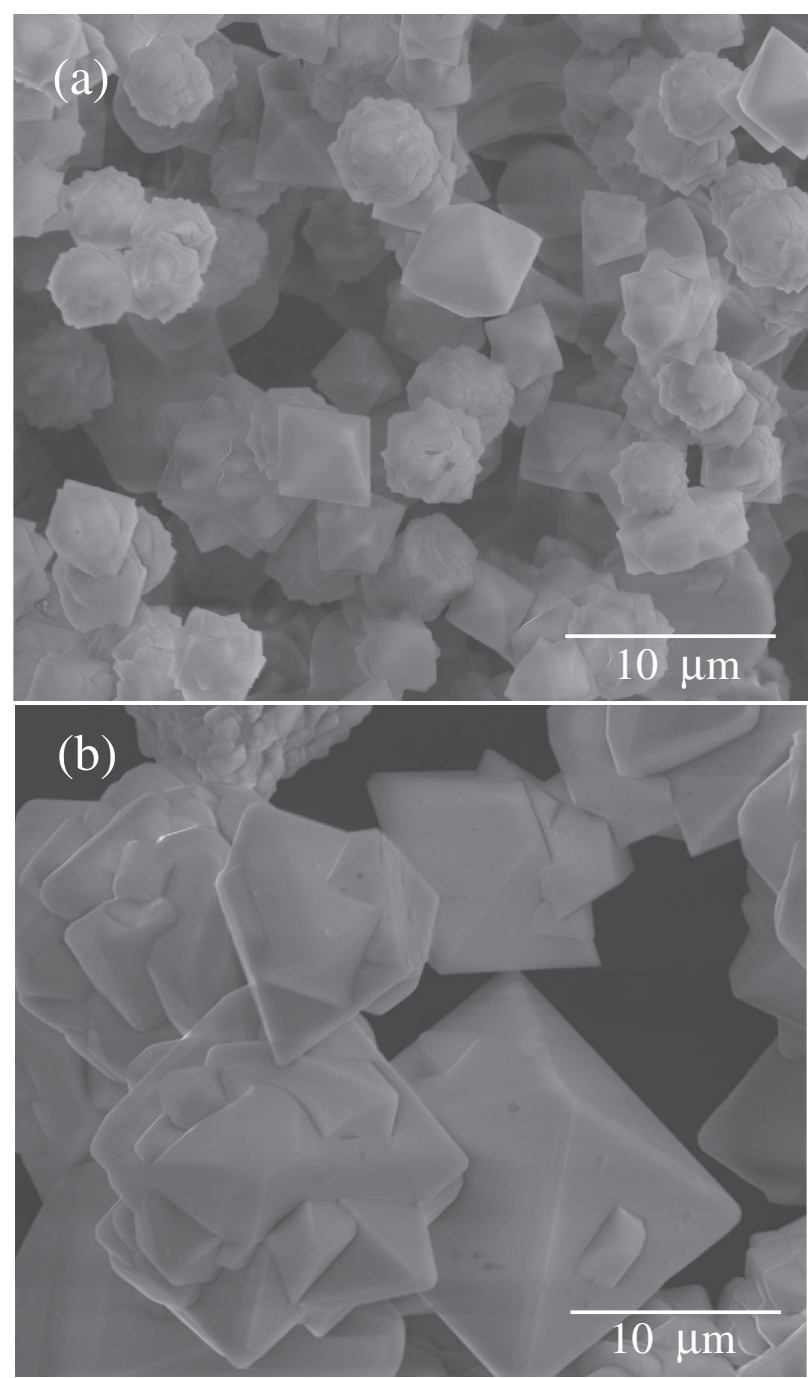

Fig. 3 SEM images of the $\mathrm{ZnO}$ crystals synthesized via thermal evaporation of $\mathrm{ZnF}_{2}$ powder at (a) $1150^{\circ} \mathrm{C}$ and (b) $1300^{\circ} \mathrm{C}$.

Any peaks from secondary phases are not observed in the XRD patterns.

EDX measurement was performed to investigate the component of the product. As shown in Fig. 2(b), only $\mathrm{Zn}$ and $\mathrm{O}$ elements are observed in the EDX spectrum. Any other elements except $\mathrm{Zn}$ and $\mathrm{O}$ are not detected, which confirms that the product is pure $\mathrm{ZnO}$.

The SEM and XRD results show that the octahedralshaped $\mathrm{ZnO}$ crystals take wurtzite crystal structure rather than zinc-blend crystal structure. There are two growth mechanisms for the formation of $\mathrm{ZnO}$ tetrapods. One is that four wurtzite legs grow on the octahedral-shaped nucleus with zinc-blend crystal structure. ${ }^{11)}$ The other is that four wurtzite legs are extended from the octahedral-shaped nucleus with wurtzite crystal structure. ${ }^{12)}$ The two tetrapod growth mechanism is still under debate. However, the present experimental observation gives evidence that $\mathrm{ZnO}$ octahedron tends to take a wurtzite crystal structure. This supports the latter growth mechanism.

According to the latter mechanism, octahedral-shaped $\mathrm{ZnO}$ nucleus is composed of eight wurtzite crystals. The crystals have a tetrahedral shape. Each tetrahedral-shaped crystal consists of three $\{11 \overline{2} 2\}$ pyramidal facets and one (0001) 


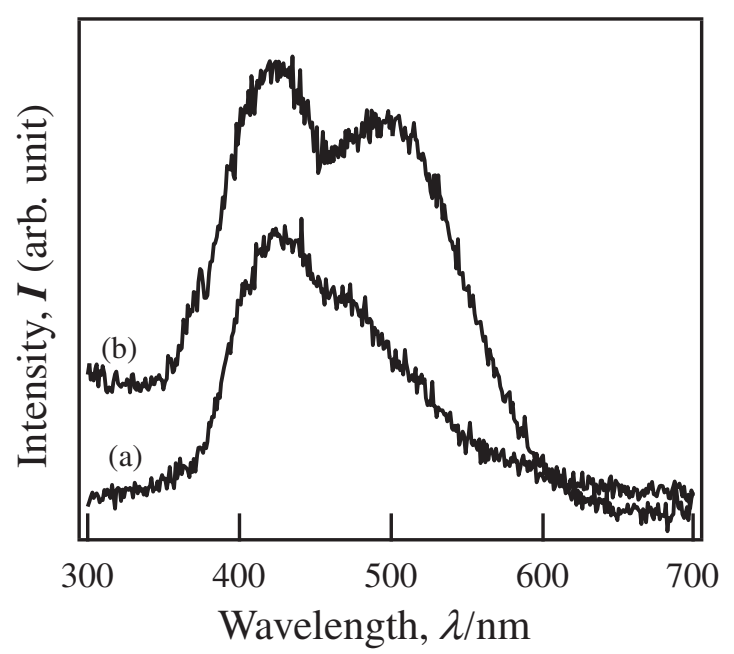

Fig. $4 \mathrm{CL}$ spectra of the $\mathrm{ZnO}$ product prepared via thermal evaporation of $\mathrm{ZnF}_{2}$ powder at (a) $1150^{\circ} \mathrm{C}$ and (b) $1300^{\circ} \mathrm{C}$.

basal facet. The eight pyramidal crystals are connected to each other at the center to form an octahedron. Thus, the surfaces of the octahedron is defined by all basal planes.

Figures 3(a) and 3(b) show the SEM images of $\mathrm{ZnO}$ crystals synthesized at 1150 and $1300^{\circ} \mathrm{C}$, respectively. As shown in Fig. 3, the $\mathrm{ZnO}$ crystals with well-defined octahedral shape are also observed for the product synthesized at an elevated temperature (up to $1300^{\circ} \mathrm{C}$ ). However the average size of the crystals increases from 4 to $10 \mu \mathrm{m}$.

Figure 4 shows the room temperature CL spectra measured for the octahedral-shaped $\mathrm{ZnO}$ crystals prepared at (a) $1150^{\circ} \mathrm{C}$ and (b) $1300^{\circ} \mathrm{C}$. Two emission peaks at around 430 (violet-blue emission) and $460 \mathrm{~nm}$ (blue emission) are observed. ${ }^{13,14)}$ It is well known that visible emission in $\mathrm{ZnO}$ crystals is related to the structural defects such as oxygen vacancies and zinc interstitials. According to the previous studies, the emission at around $430 \mathrm{~nm}$ is thought to be attributed to the electron transition from the shallow donor level of zinc interstitials to the valence band. ${ }^{13)}$ The reason is that the energy gap from the top of the valence band to the energy level of the zinc interstitial is $2.9 \mathrm{eV}$. The emission at around $460 \mathrm{~nm}$ is considered to be originated from the surface defect levels associated with oxygen vacancies. ${ }^{15)}$ As shown in Fig. 4, the intensity of the emission at around $460 \mathrm{~nm}$ significantly increases with the increase in the temperature, which suggests that the $\mathrm{ZnO}$ crystals prepared at a higher temperature have a large fraction of oxygen vacancies. The increase in the intensity of the emission at around $460 \mathrm{~nm}$ with the temperature might be related to the increase in the crystal size. Gao et al. have reported that the intensity of oxygen vacancy-related emission increases with the grain size of $\mathrm{ZnO}$ crystals. ${ }^{16)}$ On the other hand, near-band edge emission was not observed in the present experiment. It is well known that the oxygen vacancy in $\mathrm{ZnO}$ crystals can be easily produced at high temperature during the process of synthesizing $\mathrm{ZnO}$ crystals. In the present experiment, the synthetic temperature was considerably high, resulting in the high amount of defects. It is supposed that this leads to the quenching of near-band edge emission.

\section{Conclusion}

Octahedral-shaped $\mathrm{ZnO}$ crystals were synthesized by thermal evaporation without catalysts or additives. The synthetic process was carried out using $\mathrm{ZnF}_{2}$ powder as a source material in air. The octahedral-shaped $\mathrm{ZnO}$ crystals took a wurtzite crystal structure, which supports the growth mechanism of $\mathrm{ZnO}$ tetrapods based on the assumption that four legs grow over wurtzite-structured octahedral nucleus. It is supposed that the octahedral-shaped $\mathrm{ZnO}$ crystal consists of four (0001) surfaces and four (0001) surfaces. The octahedral-shaped $\mathrm{ZnO}$ crystals showed two emission peaks at 430 and $460 \mathrm{~nm}$ in the room temperature CL spectrum, which can come from zinc interstitials and oxygen vacancies. The intensity of the emission at around $460 \mathrm{~nm}$ increased with the synthetic temperature.

\section{REFERENCES}

1) X. H. Wang, R. B. Li and D. H. Fan: Appl. Surf. Sci. 257 (2011) 29602964.

2) E. S. Jang, J. H. Won, Y. W. Kim, Z. Cheng and J. H. Choy: J. Solid State Chem. 183 (2010) 1835-1840.

3) C. Xu, B. S. Kim, J. H. Lee, M. Kim, S. W. Hwang, B. L. Choi, E. K. Lee, J. M. Kim and D. Whang: Mater. Lett. 72 (2012) 25-28.

4) S. H. Mousavi, H. Haratizadeh and P. W. de Oliveira: Mater. Lett. 70 (2012) 86-88.

5) F. Giovannelli, G. Rajonson, J. Wolfman and F. Delorme: Mater. Lett. 107 (2013) 194-196.

6) K. Kametani, H. Imamoto and S. Fujita: Physica E 32 (2006) 33-36.

7) R. Guo, J. Nishimura, M. Higashihata, D. Nakamura and T. Okada: Appl. Surf. Sci. 254 (2008) 3100-3104.

8) A. Umar, E. K. Suh and Y. B. Hahn: Solid State Commun. 139 (2006) $447-451$.

9) J. M. Jang, C. R. Kim, H. Ryu, M. Razeghi and W. G. Jung: J. Alloy. Compd. 463 (2008) 503-510.

10) N. Huang, M. W. Zhu, L. J. Gao, J. Gong, C. Sun and X. Jiang: Appl. Surf. Sci. 257 (2011) 6026-6033.

11) M. Shiojiri and C. Kaito: J. Cryst. Growth 52 (1981) 173-177.

12) Y. Dai, Y. Zhang and Z. L. Wang: Solid State Commun. 126 (2003) 629-633.

13) P. K. Samanta and P. R. Chaudhuri: Mater. Lett. 68 (2012) 510-512.

14) J. Zheng, Z. Jiang, Q. Kuang, Z. Xie, R. Huang and L. Zheng: J. Solid State Chem. 182 (2009) 115-121.

15) C. Xu, D. Qin, H. Li, Y. Guoa, T. Xu and H. Li: Mater. Lett. 58 (2004) 3976-3979.

16) W. Gao, Z. W. Li, R. Harikisun and S.-S. Chang: Mater. Lett. 57 (2003) 1435-1440. 